\title{
Programmable, Transform-Limited Pulses from a Terahertz Quantum Cascade Laser
}

\author{
Iman Kundu, David R. Bacon, Paul Dean, Lianhe Li, Edmund H. Linfield, A. Giles Davies,
} and Joshua R. Freeman*

Cite This: ACS Photonics 2020, 7, 2423-2428

Read Online

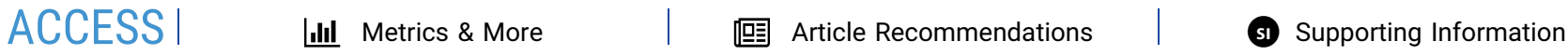

ABSTRACT: We report programmable control of pulses from a quantum cascade laser. A programmable arbitrary waveform generator is used to control the emission of the lasers by gain-switching. Quantum cascade lasers with both multimode and single-mode emission are studied. Laser emission is coherently detected using a phase-resolved injection seeding technique based on time domain spectroscopy. For the single-mode laser, pulse widths of 500-4000 ps are demonstrated, with time-bandwidth products remaining close to, or below, unity for all pulse widths. Additionally, sequences of two pulses are demonstrated, where the pulse widths, delay, and amplitude of each of the pulses are programmed electronically. A range of pulse parameters are explored. This approach allows for the generation of

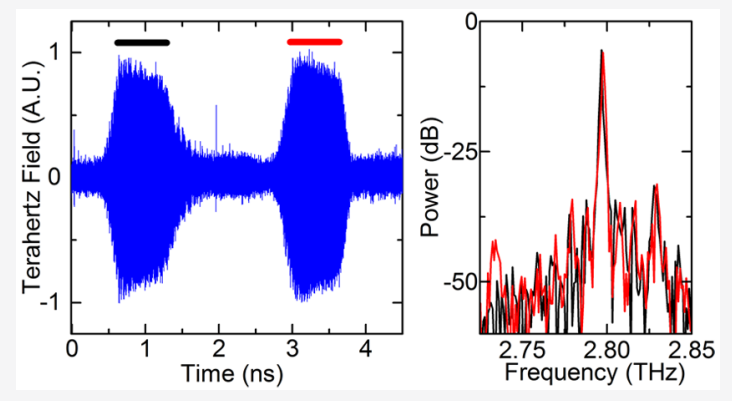
arbitrary terahertz pulse sequences, which will enable coherent multidimensional spectroscopy and applications requiring optimized pulse shapes.

KEYWORDS: optical pulses, programmable control, injection seeding, coherent detection, semiconductor lasers, terahertz (THz)

hort, powerful, narrowband pulses of electromagnetic $\checkmark$ radiation are used in many areas of science and technology to study materials. This includes pulses from visible and nearinfrared (NIR) lasers that are used to control quantum systems coherently, leading to techniques such as Rabi flopping, Hahn echo, and coherent multidimensional spectroscopy. In some areas, the use of coherent pulses has become very advanced, for example in NMR, exploited in magnetic resonance imaging scanners. Here, shaped pulses are routinely used to optimize signals, reduce artifacts, and give scientists and engineers the ability to optimize measurements for a particular characteristic. $^{1}$

The use of shaped pulses has not been widely used outside of the radio-frequencies that are typically used for NMR. This is because of the difficulty in generating powerful narrowband pulses at higher frequencies with sufficient control. Despite this, there are some exciting proposals for shaped pulses at higher frequencies. ${ }^{2-4}$ This difficulty is partly due to the shorter characteristic time scales but also the lack of mature sources in the terahertz $(\mathrm{THz})$ and infrared (IR) regions. In recent years, however, a number of mid-IR and terahertz sources have been developed that can generate and detect pulses and continuous wave radiation. Many of the most widely used sources are driven by femtosecond pulses from laser oscillators and amplifiers. These sources have many advantages, including broad bandwidths, coherent detection, and the ability to generate high peak fields. ${ }^{5}$ Techniques to shape these pulses using waveguides have also been investigated. ${ }^{6,7}$ These types of sources have been successfully applied to multidimensional spectroscopy, enabling materials to be studied at ultrafast time scales in this part of the spectrum. ${ }^{8-10}$ These techniques have mostly been applied to samples, typically solid-state, that require ultrafast transients, making generation by femtosecond lasers ideal. Other condensed matter samples have narrower spectral features, as do gases, and therefore require longer pulses that are more spectrally narrow. For these samples narrowband laser pulses are more suitable.

Pulses of light from lasers can be generated by a variety of techniques such as modelocking, Q-switching, and gainswitching. ${ }^{11}$ Each has advantages; the shortest pulse will typically be generated by mode-locking, for example. Qswitching is most applicable for optically pumped lasers with long upper state lifetimes, whereas for electrically pumped semiconductor lasers, gain-switching is the most suitable choice for generating pulses with easily controllable amplitudes and durations. This is particularly true for quantum cascade lasers (QCLs), ${ }^{12}$ which have recently become the dominant laser source in the mid-IR region $(3-15 \mu \mathrm{m})$, with multiwatt

Received: March 23, 2020

Published: July 1, 2020 
emission at room temperature, ${ }^{13}$ and increasingly the $\mathrm{THz}$ region $(2-5 \mathrm{THz}){ }^{14}$ where peak powers of $2.4 \mathrm{~W}$ have been demonstrated. $^{15}$

Gain-switching is ideally suited to QCLs because they are intrinsically fast devices, with modulation speeds of up to 35 GHz. ${ }^{16}$ This modulation capability is due to the short upper state and gain recovery lifetimes present in these unipolar lasers, which is around 20 ps. ${ }^{17-19}$ This short lifetime has also made the realization of mode-locking in QCLs challenging, ${ }^{20}$ tending to favor comb formation, ${ }^{21}$ but short pulses from midinfrared cascade lasers have recently been produced. ${ }^{22}$ The fast modulation possible with QCLs indicates that pulse durations below 0.1 ns should be possible by gain-switching. The realization of programmable pulses from QCLs operating with these durations could enable a number of applications across the mid-IR and THz. These include coherent multidimensional spectroscopy of gases that has great potential for understanding both chemical reaction dynamics and also fundamental physics. These techniques have recently been developed in the near-infrared and visible parts of the spectrum, ${ }^{23}$ with further potential for development in the $\mathrm{THz}$ and mid-IR regions, where many gases have strong active absorption features.

In this work we demonstrate how a QCL operating in the $\mathrm{THz}$ region can be combined with a gain-switching pulse from an arbitrary function generator and injection seeding to produce shaped, near-transform-limited pulses that can be sampled coherently. ${ }^{24,25}$

\section{EXPERIMENTAL SECTION}

A terahertz QCL active region was grown using molecular beam epitaxy on a semi-insulating GaAs substrate. The growth started with a 250 -nm-thick GaAs buffer layer then a $300-\mathrm{nm}$ thick $\mathrm{Al}_{0.5} \mathrm{Ga}_{0.5}$ As etch stop layer followed by a 700 -nm-thick GaAs buried contact layer, n-doped with $\mathrm{Si}$ at $2 \times 10^{18} \mathrm{~cm}^{-3}$. The QCL active region was grown next and consisted of 92 repetitions of alternating layers of $\mathrm{Al}_{0.15} \mathrm{Ga}_{0.85} \mathrm{As} / \mathrm{GaAs}$ in the following sequence (starting from the injector): $8.6 / 3 / 7.1 / 3$ / 17/3/14.5/4/10.1/0.5/16.2/1/12.9/2/11.8/3/9.5/3 nm (barriers in italics). The $17-\mathrm{nm}$-wide well (underlined) was ndoped with $\mathrm{Si}$ at $2 \times 10^{16} \mathrm{~cm}^{-3}$. This design was scaled from ref 26. Finally, a 50-nm-thick n-doped GaAs layer, with a $\mathrm{Si}$ doping density of $5 \times 10^{18} \mathrm{~cm}^{-3}$, forming the top electrical contact was grown. QCLs with $150-\mu \mathrm{m}$-wide semi-insulating surface plasmon waveguides were processed, and a $2.5-\mathrm{mm}-$ long laser was cleaved and soldered onto a copper heat sink.

The device was characterized with quasi-DC pulses $(10 \mathrm{kHz}$ repetition rate) in a continuous flow cryostat to measure the light-current-voltage relationship using a pyroelectric detector. A Fourier transform infrared spectrometer was used to measure the spectra. The electric field from the QCL was then coherently measured using a phase-resolved injection seeding technique based on time-domain spectroscopy. The experimental setup used for injection seeding and coherent detection is illustrated in Figure 1. Femtosecond optical pulses with a wavelength of $800 \mathrm{~nm}$ and a repetition rate of $80.3 \mathrm{MHz}$ were generated using a Ti:sapphire laser. A fraction of the power from these pulses was used to trigger a programmable arbitrary function generator, using a fast photodiode, then amplified to generate fast gain-switching (GS) pulses.

The GS pulses were combined with quasi-DC pulses using a bias-tee to electrically drive the QCL above the laser threshold. The remaining $800 \mathrm{~nm}$ pulse was split to form a pump beam

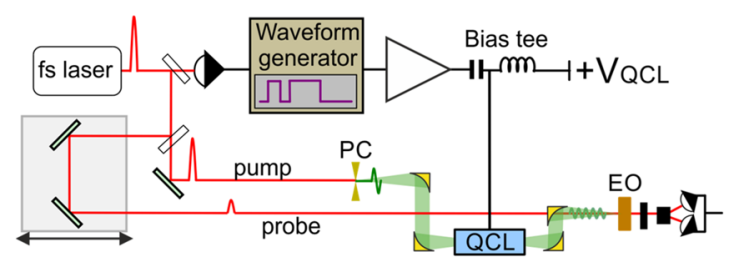

Figure 1. Schematic diagram of the experimental setup used to measure the phase-resolved electric field from the terahertz QCL. The femtosecond ( $\mathrm{fs}$ ) laser is used to trigger an arbitrary waveform generator; the pulses are amplified and combined with the quasi-DC voltage $V_{\mathrm{QCL}}$, using a bias tee, then used to drive the QCL mounted in a cryostat. The remainder of the fs pulses are split to form a pump and a probe beam. The pump beam generates a broadband $\mathrm{THz}$ seed pulse on a photoconductive (PC) switch that is focused into a QCL cavity. The QCL field is coherently sampled by the time-delayed probe beam using a $\mathrm{ZnTe}(\mathrm{EO})$ crystal.

and a probe beam. The pump was focused onto a photoconductive antenna to generate broadband terahertz pulses that were used to injection seed the QCL, with typically 5-10\% coupling efficiency. ${ }^{27}$ The terahertz field emitted from the laser was sampled using an electro-optic crystal with the time-delayed probe beam. The GS pulses from the arbitrary function generator were programmed to construct bespoke pulse shapes. The arbitrary function generator allows waveforms to be specified with a time resolution of $62.5 \mathrm{ps}$ (time difference between two points in the waveform).

\section{RESULTS}

Programmable Emission from a Multimode QCL. The peak steady-state output power of the QCL was measured as $14.3 \mathrm{~mW}$ from a single facet at a heat sink temperature of $10 \mathrm{~K}$ when the device was driven at 1.6 A using quasi-DC pulses [Figure 2(a)]. Light-current characteristics were also

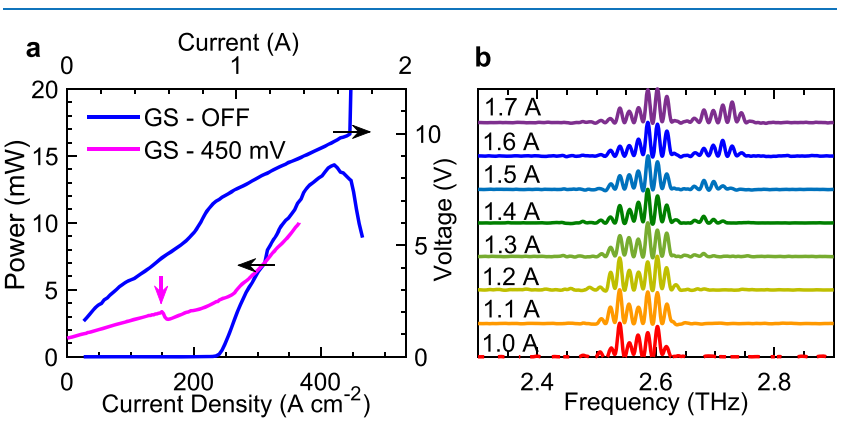

Figure 2. Experimental data from the QCL acquired in pulsed mode using a pulse train with a $20 \%$ duty cycle. (a) Light-current-voltage relationship without and with GS pulses at heat sink temperatures of 10 and $25 \mathrm{~K}$, respectively. The current contribution from the GS pulse is not included on the $x$-axis. (b) Emission spectra at different drive currents measured at $10 \mathrm{~K}$.

measured when the GS electrical pulses were combined with the quasi-DC pulses, but without seeding. A square-shaped GS pulse with an amplitude of $450 \mathrm{mV}$ and a duty cycle of $40 \%$ (pulse period of $12.45 \mathrm{~ns}$ ) was amplified then combined with the quasi-DC using a bias-tee. The GS pulse drives the QCL above the lasing threshold at a lower quasi-DC current and results in a localized maximum in the light-current measurements. The extra current provided by the GS pulses was not measured. 
The QCL spectra were measured using quasi-DC pulses, with emission frequencies between 2.54 and $2.61 \mathrm{THz}$ recorded when the current was varied between 1 and $1.4 \mathrm{~A}$ at a heat sink temperature of $10 \mathrm{~K}$ [Figure 2(b)]. The emission extended to $2.73 \mathrm{THz}$ when the current was increased to $1.5-$ $1.7 \mathrm{~A}$.

The broadband seed pulse was then added, and the QCL emission was coherently sampled at a heat sink temperature of $25 \mathrm{~K}$. The same square GS pulse, together with a quasi-DC current pulse of $0.56 \mathrm{~A}$, was used to drive the QCL [magenta arrow in Figure 2(a)]. The terahertz electric field was measured as a function of time, and a steady-state emission was observed after $\sim 500$ ps. The characteristic amplitude modulation in the terahertz field arises from the beating between the laser modes.

The temporal characteristics of the QCL emission were controlled by programming the shape of the GS pulses. The duration of the ON time of the QCL was reduced from $5 \mathrm{~ns}$ [Figure 3(a)] to 1190 ps [Figure 3(b)]. The switch-on and

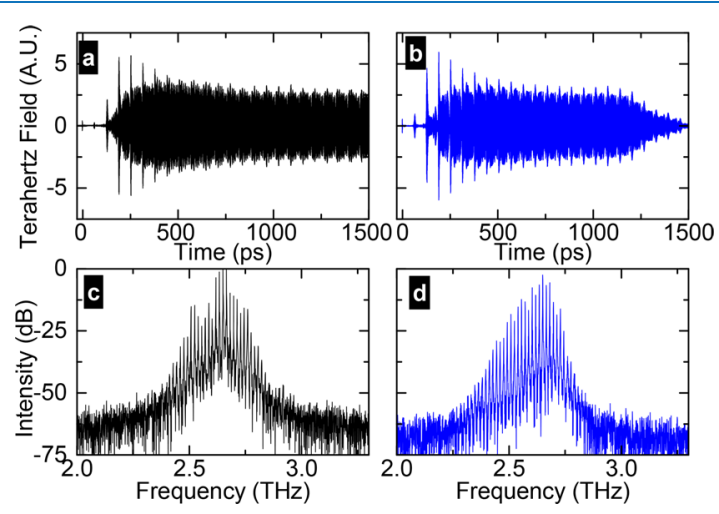

Figure 3. Experimentally measured terahertz field and spectra from the QCL at a heat sink temperature of $25 \mathrm{~K}$. Measured terahertz field using a GS pulse with a (a) 5-ns- and (b) 1187.5-ps-long ON state. Normalized emission spectra calculated from Fourier transform of the time domain terahertz field with an ON state of (c) 5 ns and (d) 1187.5 ps.

steady-state emission characteristics using the shorter pulse shape are very similar to that shown in Figure 3(a). The terahertz field from the QCL decays between 1200 and 1550 ps due to the electrical pumping switching off, as expected.

Because the measurement is coherent, the emission spectrum of each pulse can be calculated from the Fourier transform of the terahertz electric field [Figure 3(c,d)]. The spectral power is distributed over multiple longitudinal modes for both pulse shapes. The shorter duration of the GS pulse modifies the spectrum slightly, with relatively less power at frequencies greater than $2.72 \mathrm{THz}$ because the laser is operating at high currents for a proportionally shorter time owing to the finite rise/fall time.

Frequency Switchable Single-Mode QCL. For many applications narrowband pulses with transform-limited line widths are desirable. To achieve this, the emission properties of the QCL were modified using a finite defect site photonic lattice to promote single-mode operation. ${ }^{28}$ Single-mode emission eliminates the complex temporal amplitude modulation from the beating of multiple modes.

The QCL was patterned using focused ion beam milling to form a finite defect site photonic lattice with a periodicity of $16.015 \mu \mathrm{m}$ and a $50 \%$ duty cycle. A $\pi$-phase-shifted defect of length $8.07 \mu \mathrm{m}$ was also included in the center of the lattice structure, and the entire lattice was displaced from the center of the QCL cavity by $22.5 \mu \mathrm{m}$.

The emission properties of the QCL after the patterning of the photonic lattice were simulated using transfer matrices. A switchable single-mode emission at 2.731 and $2.803 \mathrm{THz}$ with a blue shift in frequency with an increase in the gain in the cavity was predicted by the simulation.

The light-current-voltage characteristic and spectra from the device was measured after the patterning of the photonic lattice. The output power from the device decreased slightly after the patterning to a peak value of $11.1 \mathrm{~mW}$ per facet at a heat sink temperature of $10 \mathrm{~K}$ and when the device was driven at 1.24 A using quasi-direct current pulses [Figure 4(a)].

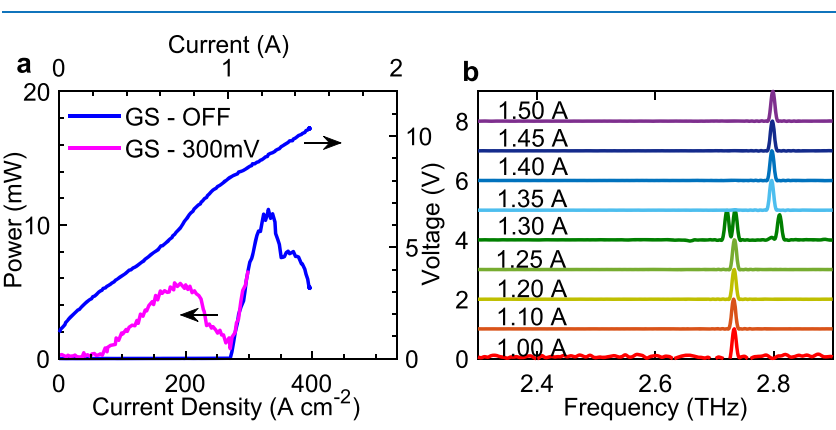

Figure 4. Experimental data from the QCL after patterning with a photonic lattice, acquired in pulsed mode using a pulse train with a $20 \%$ duty cycle. (a) Light-current-voltage relationship without and with GS pulses at heat sink temperatures of 10 and $25 \mathrm{~K}$, respectively. (b) Emission spectra at different drive current measured at $25 \mathrm{~K}$.

The QCL spectra indicate single-mode emission at 2.735 $\mathrm{THz}$ when the current is varied between 1 and $1.25 \mathrm{~A}$ and at a heat sink temperature of $25 \mathrm{~K}$. A mode hop to $2.797 \mathrm{THz}$ is recorded when the current increases to 1.35-1.50 A [Figure 4(b)].

The terahertz field from the modified QCL was sampled using the same phase-resolved injection seeding technique. The QCL was electrically driven using the same square-shaped GS pulses with an amplitude of $300 \mathrm{mV}$ and a duty cycle of $40 \%$ (pulse period of $12.45 \mathrm{~ns}$ ), which were amplified and combined with quasi-DC pulses. The amplitude of $300 \mathrm{mV}$ was chosen to allow access to the full operating range of the QCL by controlling the quasi-DC bias. The terahertz field was measured at different quasi-DC biases [Figure 5(a)], and the spectra were calculated by Fourier transform of the measured terahertz field [Figure 5(b)]. Emission at $2.735 \mathrm{THz}$ was measured at terminal voltages of 5-9 V. The time required for the QCL emission to stabilize [Figure 5(a) inset] increases for these biases. Increasing the bias to $10 \mathrm{~V}$ resulted in dual-mode operation and reduced the stabilization time. When the bias is increased to $11 \mathrm{~V}$, the emission frequency hops to $2.797 \mathrm{THz}$, and a further increase in the stabilization time was recorded. This variation in switch-on delay has been discussed in ref 25, where it was shown the delay depends nontrivially on the laser cavity transmission function. The emission frequencies calculated from the time-domain measurements agree well with the spectra shown in Figure 4(b).

Although a steady-state single-mode emission was observed for all terminal voltages except at $10 \mathrm{~V}$, a multimode emission was observed before the QCL emission stabilized. The stabilization time also decreased during the mode hop. Both 

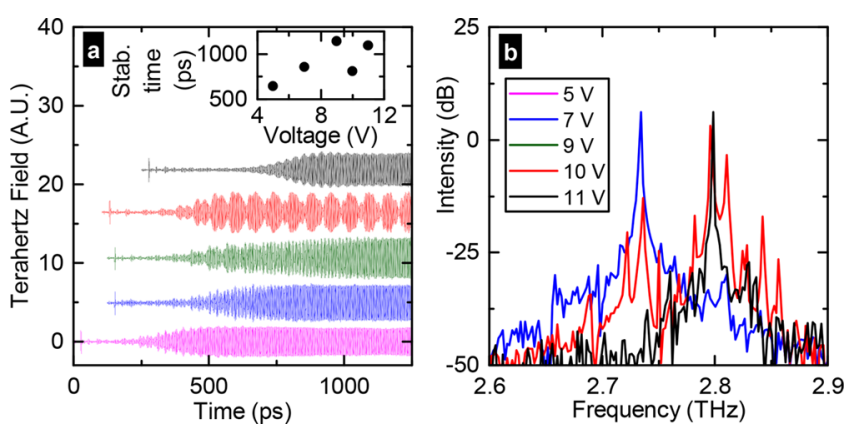

Figure 5. Experimentally measured terahertz field and spectra from the QCL after patterning with a photonic lattice. Data recorded at a heat sink temperature of $25 \mathrm{~K}$ using a GS pulse with 5-ns-long ON state and an amplitude of $300 \mathrm{mV}$. (a) Terahertz field measured at different QCL terminal voltage. Inset: Variation of the stabilization time as a function of the terminal voltage. (b) Emission spectra calculated from Fourier transform of the time domain terahertz field when the QCL terminal voltages are 7,10 , and $11 \mathrm{~V}$.

of these observations agree with results from a Vernier tunable QCL discussed in ref 25 . In the subsequent measurements steady-state emission at 6 and $11 \mathrm{~V}$ was used for programming the pulse envelope from the QCL. First, the duration of the $\mathrm{ON}$ state of a single pulse was varied systematically.

Programming the Duration of a Terahertz Pulse. The width of the terahertz pulse was varied by modifying the duration of the ON state of the GS pulses from 875 to 3875 ps, with the same amplitude as Figure 5.

The measured terahertz fields are shown in Figure 6(a). The switch-on delay was measured to be 580 ps when the QCL

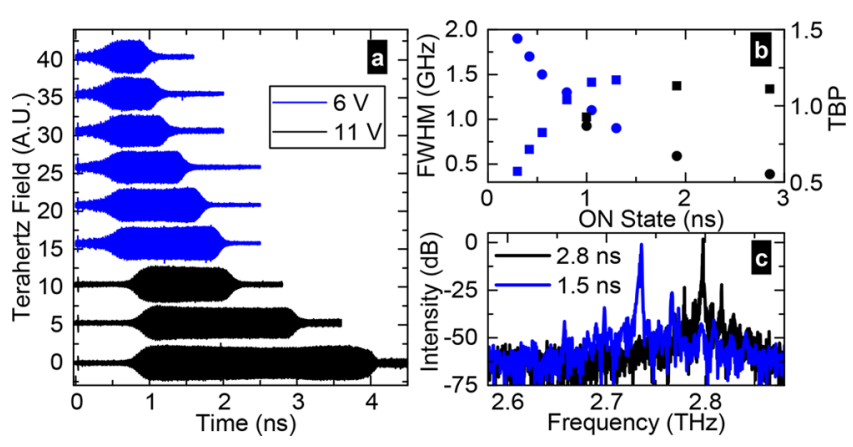

Figure 6. Measured terahertz field and spectra from the QCL after patterning with a photonic lattice. Data were recorded at a heat sink temperature of $25 \mathrm{~K}$. (a) Terahertz field measured at QCL terminal voltages of $6 \mathrm{~V}$ (blue) and $11 \mathrm{~V}$ (black) when the pulse widths are varied in the range 0.4-2.8 ns. (b) Variation of the fwhm (circles) and time-bandwidth product (TBP) (squares). (c) Emission spectra calculated from Fourier transform of the time domain data when the steady-state width of the terahertz field is $1.5 \mathrm{~ns}(6 \mathrm{~V})$ and $2.8 \mathrm{~ns}(11$ V).

terminal voltage is at $6 \mathrm{~V}$ (blue). This increased to $980 \mathrm{ps}$ when the terminal voltage was increased to $11 \mathrm{~V}$ (black). The time required for the terahertz photons to exit the QCL cavity and for the emission to cease was recorded to be 260 ps.

The width of the ON state (steady-state emission excluding the pulse rise and fall time, measured by $90 / 10$ criteria) was recorded to be $300,420,550,800,1050$, and 1300 ps when the QCL terminal voltage was $6 \mathrm{~V}$ and the widths of the electrical GS pulses were varied as $875,1000,1125,1375,1625$, and 1875 ps, indicating a consistent 575 ps difference, commensu- rate with the switch-on delay. The emission frequency for these pulses was calculated to be $2.735 \mathrm{THz}$ [Figure 6(c)]

Similarly, the width of the steady-state emission was measured to be 1020, 1900, and 2900 ps when the QCL terminal voltage was $11 \mathrm{~V}$ and the widths of the GS pulses were set to 2000, 2875, and 3875 ps, again showing a consistent difference of 975 ps, commensurate with the measured switch-on delay. The emission frequency for these pulses was measured to be $2.797 \mathrm{THz}$ [Figure 6(c)]. Figure 6(b) shows how the fwhm and time-bandwidth product (TBP) depend on the pulse duration. The shortest pulses have a TBP less than 1, because of the finite switch-on and switchoff times. A perfect square pulse at the transform limit would have a TBP of 1 . For the longer pulse durations, shown in Figure 6, the TBP increases slightly above 1 . Detailed analysis of the pulse shows that the frequency during the initial buildup is approximately $0.6 \mathrm{GHz}$ higher than the steady state.

These measurements illustrate how terahertz pulse parameters such as the pulse duration and emission frequency can be controlled through appropriate adjustment of the QCL terminal voltage and width of the GS pulses. For example, the emission frequency can be blue-shifted (from $2.735 \mathrm{THz}$ to $2.797 \mathrm{THz}$ ), while maintaining a 1 ns pulse width by simply increasing the QCL terminal voltage (from 6 to $11 \mathrm{~V}$ ) and the GS pulse widths (from 1700 to 2062 ps).

Programming Two Short Bursts of Terahertz Pulses. In addition to controlling the duration of a single pulse, it is also possible to generate consecutive pulses. The shape of the GS pulse was modified to form two pulses; by doing this we are able to control the pulse widths, the delay between each pulse, and the amplitude of each pulse independently.

The shape of the GS waveforms for twin pulses is nontrivial and requires careful optimization of the OFF state between the two pulses in order to preserve the phase of the injected broadband seed pulse. To this end, the amplitude of the OFF state was systematically calibrated and maintained at transparency. Once the GS waveform was programmed, the amplitude and offset of the GS pulse were fine-tuned using both the GS pulse amplitude and quasi-DC bias, respectively. This ensures a minimal terahertz field between the two pulses while preserving the phase of the seed pulse so that the terahertz field of the second pulse remains correlated with the first pulse and the femtosecond pulse that samples the electric field.

The first pulse was designed to have a 450-ps-wide ON state ( 970 -ps-wide pulses including the pulse rise and fall time). The second pulse was programmed to be generated 875 ps after the end of first pulse. In order to ensure proper seeding of the second pulse while maintaining the OFF state between the two pulses, the amplitude of the second pulse was varied systematically while monitoring the seeding coherence. Following this, the amplitude of the second pulse waveform was increased by $30 \%$ compared to the first pulse to ensure that the $\mathrm{THz}$ pulses have equal amplitudes.

The terahertz field measured using this GS pulse shape is shown in Figure 7(a). The widths of the first and the second pulses are measured to be 990 and 970 ps, agreeing closely with the designed pulse shapes. The delay between the two pulses (measured to be $\sim 2.3 \mathrm{~ns}$ ) is longer than the 875-pswide delay between the electronic waveforms, due to the switch-on delay of the QCL for the second pulse. The seeding of the QCL emission was verified by measuring the terahertz field as a function of antenna voltage at delay positions 921.04 

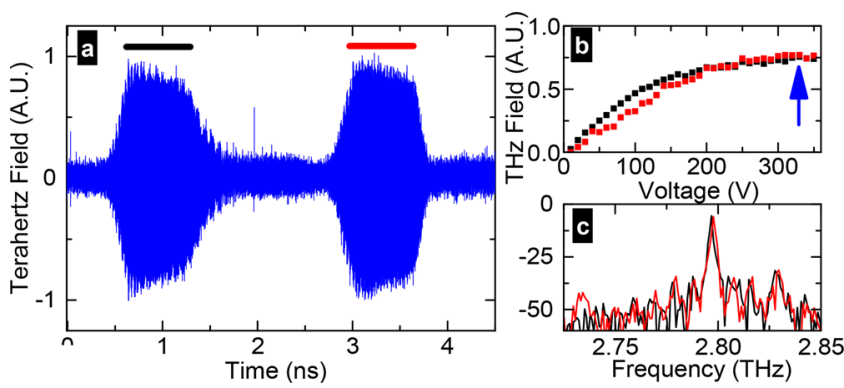

Figure 7. Two short bursts of terahertz field. Data recorded at a heat sink temperature of $25 \mathrm{~K}$. (a) Terahertz field measured at QCL terminal voltages $11 \mathrm{~V}$. (b) Variation of the amplitude of the terahertz field of both pulses at 921.04 ps (black) and 3399.30 ps (red) as a function of voltage across the photoconductive antenna. (c) Emission spectra calculated from Fourier transform of the first pulse (black) and the second pulse (red).

and 3399.30 ps [Figure $7(\mathrm{~b})$ ]. The terahertz field was measured to be saturated at the antenna voltage of $330 \mathrm{~V}$ (proportional to the seed power). This measurement confirms that the second pulse is fully seeded ${ }^{29}$ and all of the emission can be detected coherently; the two pulses are each coherent with the seed laser and therefore coherent with each other.

The frequency spectrum of the QCL emission was calculated by Fourier transform of the full time-domain data [Figure $7(\mathrm{c})$ ]. The emission frequency is at $2.797 \mathrm{THz}$ during the ON state for both the first pulse (black) and second pulse (red). During the OFF state, there is weak emission at 2.735 $\mathrm{THz}$, in accordance with the spectral changes expected as a function of bias voltage across the QCL.

To demonstrate the flexibility of this programming approach, the pulse widths, delay, and amplitude of both the pulses were varied. Figure $8(a)$ shows the second pulse width
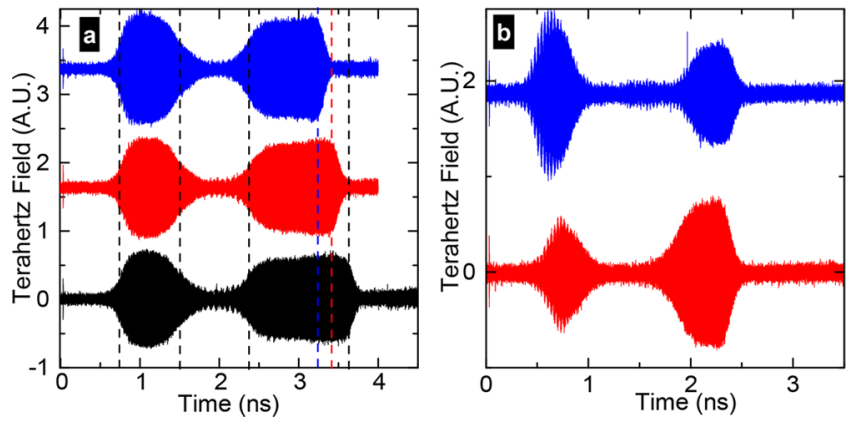

Figure 8. (a) Independent control of the duration of the second pulse by programming of the GS pulse. (b) The amplitude of the first pulse is reduced by advancing the GS trigger by 188 ps (red). The amplitude of the second pulse is reduced (blue) when the QCL voltage is reduced to $10.5 \mathrm{~V}$, the amplitude of the GS pulses is increased to $280 \mathrm{mV}$, and the trigger for the GS pulses is delayed by 63 ps.

changing by programming the GS pulse, while the delay between the pulses and width of the second pulse remains unchanged. For these measurements the quasi-DC laser bias was $11.5 \mathrm{~V}$ and the amplitude of the GS pulse was $250 \mathrm{mV}$. Data showing the variation of the first pulse width while the second is held constant are provided in the Supporting Information (Figure S2).

Finally, in Figure 8(b) we show how the amplitude of each pulse can be controlled. First, the amplitude of the second pulse is reduced, while maintaining the first (blue), then the amplitude of the first pulse is reduced while maintaining the second (red).

\section{CONCLUSION}

In conclusion, we have demonstrated an instrument that allows near-transform-limited pulses to be programed from a terahertz quantum cascade laser with injection-seeded emission so that the pulses can be coherently detected. The arbitrary function generator that was used to program the electrical pulse shapes has a resolution of 62.5 ps. By using a single-mode QCL, engineered using a finite defect photonic lattice, the pulses remain close to the transform limit for the pulse durations we are able to measure ( $\sim 4 \mathrm{~ns})$, thereby controlling the spectral width programmatically. The shortest pulse measured from this system is $\sim 650 \mathrm{ps}$ (including a pulse rise and fall time of $\sim 580$ ps) with an ON state width of $\sim 57$ ps (Figure S1). Sequences of twin pulses have also been demonstrated where the pulse widths, delay, and amplitude have been controlled electronically without the need for additional delay stages. This narrowband pulse spectrometer will find applications in the study of narrowband excitation and relaxation processes at terahertz frequencies and in coherent communications. Additionally, because the QCL emission is phase locked to the femtosecond laser clock, these narrowband pulses could be combined with broadband probe pulses which are readily generated by the femtosecond lasers, for applications requiring narrowband-pump-broadband-probe spectroscopy, for example.

\section{ASSOCIATED CONTENT}

\section{Supporting Information}

The Supporting Information is available free of charge at https://pubs.acs.org/doi/10.1021/acsphotonics.0c00458.

Additional figures showing the shortest pulses generated by this method and the control of the width of the first pulse (PDF)

\section{AUTHOR INFORMATION}

\section{Corresponding Author}

Joshua R. Freeman - School of Electronic and Electrical Engineering, University of Leeds, Leeds LS2 9JT, United Kingdom; 이이이.org/0000-0002-5493-6352; Email: j.r.freeman@leeds.ac.uk

\section{Authors}

Iman Kundu - School of Electronic and Electrical Engineering, University of Leeds, Leeds LS2 9JT, United Kingdom; (1) orcid.org/0000-0002-3564-1903

David R. Bacon - School of Electronic and Electrical Engineering, University of Leeds, Leeds LS2 9JT, United Kingdom

Paul Dean - School of Electronic and Electrical Engineering, University of Leeds, Leeds LS2 9JT, United Kingdom

Lianhe Li - School of Electronic and Electrical Engineering, University of Leeds, Leeds LS2 9JT, United Kingdom; (1) orcid.org/0000-0003-4998-7259

Edmund H. Linfield - School of Electronic and Electrical Engineering, University of Leeds, Leeds LS2 9JT, United Kingdom 
A. Giles Davies - School of Electronic and Electrical Engineering, University of Leeds, Leeds LS2 9JT, United Kingdom

Complete contact information is available at:

https://pubs.acs.org/10.1021/acsphotonics.0c00458

\section{Author Contributions}

All authors have given approval to the final version of the manuscript.

\section{Notes}

The authors declare no competing financial interest.

\section{ACKNOWLEDGMENTS}

This work was supported by the Engineering and Physical Sciences Research Council, U.K. (HyperTerahertz Programme

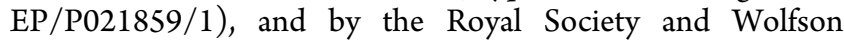
Foundation. Research data associated with this paper are openly available from The University of Leeds data repository: https://doi.org/10.5518/850.

\section{REFERENCES}

(1) Topgaard, D.; Martin, R. W.; Sakellariou, D.; Meriles, C. A.; Pines, A. Shim pulses" for NMR spectroscopy and imaging. Proc. Natl. Acad. Sci. U. S. A. 2004, 101, 17576-17581.

(2) Fillion-Gourdeau, F.; Hebenstreit, F.; Gagnon, D.; MacLean, S. Pulse shape optimization for electron-positron production in rotating fields. Phys. Rev. D: Part. Fields 2017, 96 (1), 016012.

(3) Gagnon, D.; Dumont, J.; Fillion-Gourdeau, F.; MacLean, S. Pulse shaping in the terahertz frequency range for the control of photo-excited carriers in graphene. J. Opt. Soc. Am. B 2018, 35 (12), 3021-3029.

(4) Gagnon, D.; Fillion-Gourdeau, F.; Dumont, J.; Lefebvre, C.; MacLean, S. Suppression of Multiphoton Resonances in Driven Quantum Systems via Pulse Shape Optimization. Phys. Rev. Lett. 2017, 119 (5), 053203.

(5) Terahertz Spectroscopy and Imaging; Springer, 2013; p 629.

(6) Gingras, L.; Cooke, D. G. Direct temporal shaping of terahertz light pulses. Optica 2017, 4 (11), 1416-1420.

(7) Gingras, L.; Cui, W.; Schiff-Kearn, A. W.; Ménard, J.-M.; Cooke, D. G. Active phase control of terahertz pulses using a dynamic waveguide. Opt. Express 2018, 26 (11), 13876-13882.

(8) Gunter, G.; Anappara, A. A.; Hees, J.; Sell, A.; Biasiol, G.; Sorba, L.; De Liberato, S.; Ciuti, C.; Tredicucci, A.; Leitenstorfer, A.; Huber, R. Sub-cycle switch-on of ultrastrong light-matter interaction. Nature 2009, 458 (7235), 178-181.

(9) Kampfrath, T.; Tanaka, K.; Nelson, K. A. Resonant and nonresonant control over matter and light by intense terahertz transients. Nat. Photonics 2013, 7 (9), 680-690.

(10) Tanaka, K.; Hirori, H.; Nagai, M. THz Nonlinear Spectroscopy of Solids. IEEE Trans. Terahertz Sci. Technol. 2011, 1 (1), 301-312.

(11) Siegman, A. E. Lasers; University Science Books, 1989; p 923.

(12) Faist, J. Quantum Cascade Lasers, 1st ed.; Oxford University Press: Oxford, 2013.

(13) Razeghi, M.; Zhou, W.; Slivken, S.; Lu, Q.-Y.; Wu, D.; McClintock, R. Recent progress of quantum cascade laser research from 3 to 12 (m at the Center for Quantum Devices [Invited]. Appl. Opt. 2017, 56 (31), H30-H44.

(14) Williams, B. S. Terahertz quantum-cascade lasers. Nat. Photonics 2007, 1 (9), 517-525.

(15) Li, L. H.; Chen, L.; Freeman, J. R.; Salih, M.; Dean, P.; Davies, A. G.; Linfield, E. H. Multi-Watt high-power THz frequency quantum cascade lasers. Electron. Lett. 2017, 53 (12), 799-800.

(16) Gellie, P.; Barbieri, S.; Lampin, J.-F.; Filloux, P.; Manquest, C.; Sirtori, C.; Sagnes, I.; Khanna, S. P.; Linfield, E. H.; Davies, A. G.; Beere, H.; Ritchie, D. A. Injection-locking of terahertz quantum cascade lasers up to $35 \mathrm{GHz}$ using RF amplitude modulation. Opt. Express 2010, 18 (20), 20799-20816.

(17) Bacon, D. R.; Freeman, J. R.; Mohandas, R. A.; Li, L.; Linfield, E. H.; Davies, A. G.; Dean, P. Appl. Phys. Lett. 2016, 108 (8), 081104.

(18) Green, R. P.; Tredicucci, A.; Vinh, N. Q.; Murdin, B.; Pidgeon, C.; Beere, H. E.; Ritchie, D. A. Gain recovery dynamics of a terahertz quantum cascade laser. Phys. Rev. B: Condens. Matter Mater. Phys. 2009, 80 (7), 075303.

(19) Derntl, C. G.; Scalari, G.; Bachmann, D.; Beck, M.; Faist, J.; Unterrainer, K.; Darmo, J. Appl. Phys. Lett. 2018, 113 (18), 181102.

(20) Wang, F.; Maussang, K.; Moumdji, S.; Colombelli, R.; Freeman, J.; Kundu, I.; Li, L.; Linfield, E. H.; Davies, A. G.; Mangeney, J.; Tignon, J.; Dhillon, S. S. Generating ultrafast pulses of light from quantum cascade lasers. Optica 2015, 2 (11), 944-949.

(21) Hillbrand, J.; Andrews, A. M.; Detz, H.; Strasser, G.; Schwarz, B. Coherent injection locking of quantum cascade laser frequency combs. Nat. Photonics 2019, 13 (2), 101-104.

(22) Hillbrand, J.; Beiser, M.; Andrews, A. M.; Detz, H.; Weih, R.; Schade, A.; Höfling, S.; Strasser, G.; Schwarz, B. Picosecond pulses from a mid-infrared interband cascade laser. Optica 2019, 6 (10), $1334-1337$.

(23) Bruder, L.; Bangert, U.; Binz, M.; Uhl, D.; Stienkemeier, F. Coherent multidimensional spectroscopy in the gas phase. J. Phys. B: At., Mol. Opt. Phys. 2019, 52 (18), 183501.

(24) Oustinov, D.; Jukam, N.; Rungsawang, R.; Madéo, J.; Barbieri, S.; Filloux, P.; Sirtori, C.; Marcadet, X.; Tignon, J.; Dhillon, S. Phase seeding of a terahertz quantum cascade laser. Nat. Commun. 2010, 1 $(1), 69$.

(25) Kundu, I.; Wang, F.; Qi, X.; Nong, H.; Dean, P.; Freeman, J. R.; Valavanis, A.; Agnew, G.; Grier, A. T.; Taimre, T.; Li, L.; Indjin, D.; Mangeney, J.; Tignon, J.; Dhillon, S. S.; Rakić, A. D.; Cunningham, J. E.; Linfield, E. H.; Davies, A. G. Ultrafast switch-on dynamics of frequency-tuneable semiconductor lasers. Nat. Commun. 2018, 9 (1), 3076.

(26) Wienold, M.; Schrottke, L.; Giehler, M.; Hey, R.; Anders, W.; Grahn, H. T. Low-voltage terahertz quantum-cascade lasers based on LO-phonon-assisted interminiband transitions. Electron. Lett. 2009, 45 (20), 1030-1031.

(27) Freeman, J. R.; Ponnampalam, L.; Shams, H.; Mohandas, R. A.; Renaud, C. C.; Dean, P.; Li, L.; Giles Davies, A.; Seeds, A. J.; Linfield, E. H. Injection locking of a terahertz quantum cascade laser to a telecommunications wavelength frequency comb. Optica 2017, 4 (9), $1059-1064$.

(28) Kundu, I.; Dean, P.; Valavanis, A.; Li, L.; Han, Y.; Linfield, E. H.; Davies, A. G. Frequency Tunability and Spectral Control in Terahertz Quantum Cascade Lasers With Phase-Adjusted FiniteDefect-Site Photonic Lattices. IEEE Trans. Terahertz Sci. Technol. 2017, 7 (4), 360-367.

(29) Maysonnave, J.; Jukam, N.; Ibrahim, M. S. M.; Rungsawang, R.; Maussang, K.; Madéo, J.; Cavalié, P.; Dean, P.; Khanna, S. P.; Steenson, D. P.; Linfield, E. H.; Davies, A. G.; Dhillon, S. S.; Tignon, J. Measuring the sampling coherence of a terahertz quantum cascade laser. Opt. Express 2012, 20 (15), 16662-16670. 